\title{
Retrieval of a metallic foreign body in the neck with a rare earth magnet
}

\author{
Jeremy T H Chin, Stephen J Davies, Jonathan P Sandler
}

A 30 year old man was referred by the accident and emergency (A\&E) department to the oral and maxillofacial surgery unit with small puncture lacerations on his left cheek and lower neck. The patient, a metal fitter, had been hammering on a piece of metal that shattered on impact. Local examination revealed two small lacerations (approximately $2 \mathrm{~cm}$ each) sited in the left cheek and left inferior neck region. Two discrete metal foreign bodies (approximately $1 \mathrm{~cm}$ in diameter) were detected by plain radiography (true lateral skull) (fig 1). The foreign body in the left cheek region was readily palpated through the skin and was retrieved via a small skin incision under local anaesthesia. The fragment in the neck was located in deeper subcutaneous tissues and was not readily palpable. Initial attempts to retrieve the object using surgical exploration with the aid of an image intensifier failed. The main reason for leaving metal fragments is that the removal procedure is laborious, destructive of surrounding tissues and may leave scars on the surrounding skin. The orthodontic consultant was subsequently consulted by the maxillofacial surgeons to explore the possibility of using a rare earth magnet for the retrieval of the metallic object.

Rotherham District General Hospital,

Rotherham

J T H Chin

Chesterfield and North Derbyshire Royal

Hospital, Chesterfield S44 5BL

S J Davies

J P Sandler

Correspondence to:

Mr Sandler, Consultant in Orthodontics

(JonSandler@AOL.com)

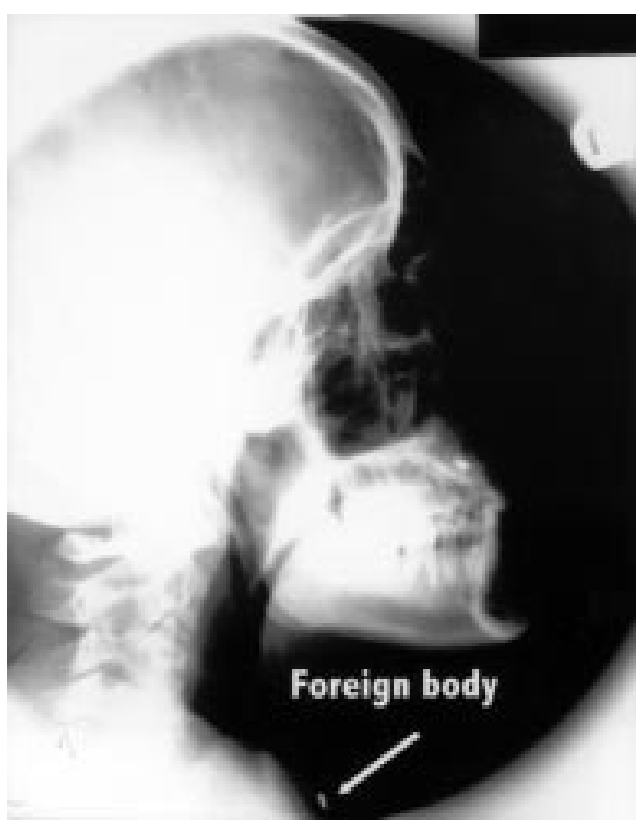

Figure 1 True lateral radiograph of face and neck.
A neodymium iron boron magnet was held over the laceration and the metal fragment was instantaneously attracted onto the surface of the magnet (fig 2). The lacerated sites were closed with Ethilon sutures and healing was uneventful.

Penetrating injuries caused by sharp metallic objects constitute a major proportion of all lacerations observed in emergency departments. ${ }^{1}$ Once embedded in soft tissues, metallic fragments, particularly when small and multiple, are difficult to detect and retrieve. Detection in vivo may be facilitated by various investigative techniques including plain radiography, xerography, computed tomography, ultrasonography and metal detectors. $^{23}$ Retrieval conventionally follows meticulous surgical exploration of the lacerated site. The metallic fragment in this report possessed sufficient iron content to permit its retrieval by a magnet held at the wound opening. Neodymium iron boron is the most powerful rare earth magnet used in dentistry, particularly in the orthodontic movement of impacted teeth. ${ }^{4}$ Developments in magnet technology have resulted in marked improvements in the flux density that, in turn, has led to greater attraction forces and concomitant reductions in size. The energy density stored per unit volume is about eight times that of traditional AlNiCo horseshoe magnets and nearly 40 times that of the "fridge decoration" type magnet. Because magnetic force obeys the inverse square law, a sufficient number of magnets must be held in close proximity to the wound opening to exert adequate retraction of the foreign body. In addition, the magnet should be carefully positioned to ensure that

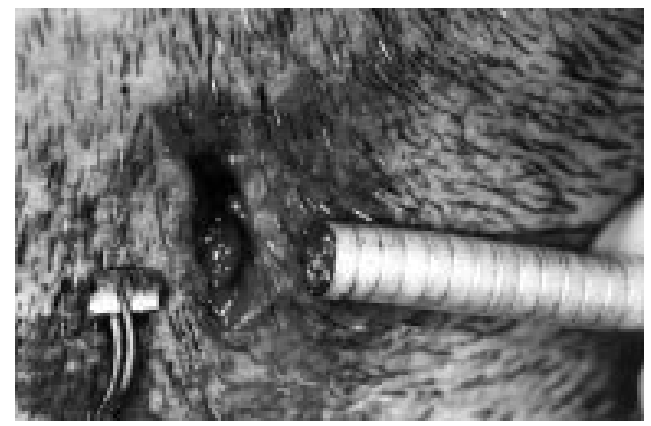

Figure 2 Foreign body held in tweezers after retrieval with magnets. 
the "trough" of maximum attraction (immediately below the magnets) runs over or close to the buried fragments. This method should ensure the safe removal of all reasonably superficial $(5-10 \mathrm{~mm})$ metal fragments that are magnetic. Accidental removal of other metallic objects such as aneurysm clips, while a theoretical possibility, is not a real risk as with magnetic resonance imaging magnets, as the "throw" of stray magnetic field is very much less. In other words the field is much more concentrated (that is, focused flashlight beam compared with the array of fluorescent tube lights). Cost of magnets obviously depends on size, coatings applied and quantity purchased but for those used in this report a price of a few pounds per magnet would be charged for small quantities. The magnets can be chemically sterilised and reused but not autoclaved as the high temperatures would destroy the magnetism.
Special training should not be needed by the $\mathrm{A} \& \mathrm{E}$ doctors provided they understand the anatomy of the area in which they are working and simple precautions are used for handling and storing the magnets. This technique of relatively atraumatic, reliable and rapid magnetic removal of metallic foreign bodies is recommended and the low cost of the magnets should ensure every A\&E department adds them to the armamentarium.

Funding: none.

Conflicts of interest: none.

1 Singer A, Hollander JE, Quinn JV. Current concepts: evaluation and management of traumatic lacerations. $N$ Engl $\mathcal{F}$ Med 1997;337:1142-8.

2 Ginsburg MJ, Ellis GL, Flom LL. Detection of soft tissue foreign bodies by plain radiography, xerography, computed tomography and ultrasonography. Ann Emerg Med 1990; 19:701-3.

3 Abe K, Nakarnatsu K, Beppu K, et ak. Use of intraoperative ultrasonography to detect a small foreign body in the soft tissues of the upper lip. Br Dent f 1994;177:292-4.

4 Sandler PJ, Meghji S, Murray AM, et al. Magnets and orthodontics. Br f Orthod 1989;16:243-9.

\title{
Circ.com-plications
}

\author{
J E Kennedy
}

\author{
Accident and \\ Emergency \\ Department, Royal \\ Hampshire County \\ Hospital, Winchester \\ Correspondence to: \\ Dr Kennedy, Senior House \\ Office, Department of \\ Surgery, Royal Hampshire \\ County Hospital, Romsey \\ Road, Winchester, \\ Hampshire SO22 5DG
}

A 20 year old single man presented to the accident and emergency department after failing in an attempt to circumcise himself. He had previously normal anatomy, and a fully retractile foreskin. He had been "browsing" on the internet and had found a web site ${ }^{1}$ with written and pictorial instructions for self circumcision techniques. Using a non-sterile craft scalpel and lignocaine (lidocaine) spray, which were "to hand", he followed these to the letter. On arrival in the department, the patient was in considerable pain, but was haemodynamically stable. There was evidence of venous haemorrhage and examination revealed a ragged incision around the outer preputial skin at the level of the corona, a small plastic "guard" (fig 1) that had been fashioned to protect the glans was also in situ.

A penile block combining lignocaine and bupivacaine allowed appropriate inspection, and circumcision was indicated. This was completed by the duty surgeon, and prophylactic antibiotics were prescribed in view of the less than sterile conditions. He was covered for tetanus. Review at one week showed the wound to be healing without complication.

Inspection of the web site ${ }^{1}$ shows a vast amount of information on circumcision, suggesting improvement in sexual function and describing the procedure variously as "blood-

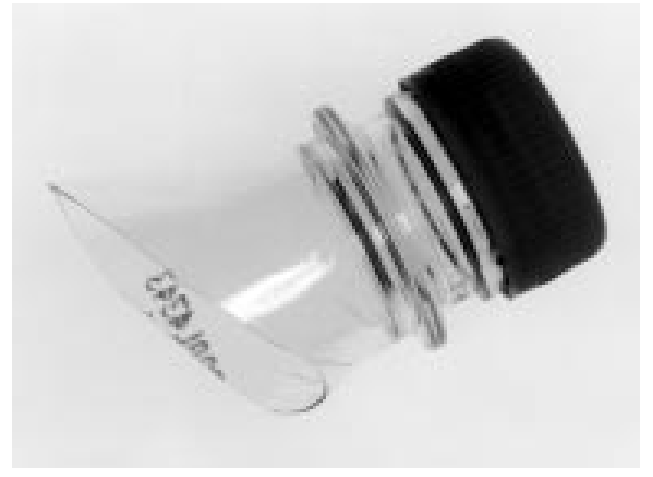

Figure 1 Small plastic "glans guard".

less", "painless", and "easy". Unfortunately the patient was reluctant to comply with long term follow up to assess any improvement! There are reported links between self castration and psychosis. ${ }^{2}$ In this event there was no evidence of psychiatric illness and the patient was not referred on. This intriguing case could be considered as a circuitous route to bypass NHS waiting lists.

My thanks to Mr Hugo Dowd for his suggestions and critical review of the manuscript.

1 www.circlist.org/

2 McGovern SJ. Self-castration in a transsexual. 7 Accid Emerg Med 1995;12:57-8. 


\title{
An unusual diagnosis for a swollen limb
}

\author{
Mark Sheldon Lloyd
}

A 30 year old black African Christian missionary lady presented to our accident and emergency department with a mildly swollen left arm. She had left Liberia 11 months before coming to the UK and had been in this country for two months. Her first attendance was a GP referral to the medical on call team. Complaints were an itchy left arm, which had become red over a month. Her symptoms had not resolved with antihistamines or other medication for allergy. There were no other symptoms at this time and she appeared well with no lymphadenopathy or neurovascular deficit in the affected arm. Her breast and chest examination were normal as was her chest radiograph and on investigation there was a mild eosinophilia. All other blood tests were normal including a malaria and sickle cell screen. A working diagnosis of an axillary vein thrombosis was made. She was given low molecular weight heparin while an ultrasound scan was arranged. She was well and the physicians discharged her with outpatient follow up. The next day she attended casualty with pain at rest in the same arm, which was gradually getting worse. The arm was hot, tender and swollen, but there was no pitting oedema or neurovascular changes. She still had full function of the arm. An emergency ultrasound scan did not confirm the presence of an axillary vein thrombosis. However, a serum sample was taken for possible parasitic infection. There were no new findings on examination. Two weeks later, her left arm had become grossly swollen from the fingertips to the shoulder, with loss of function from the elbow downwards. She was now unable to raise her arm against gravity and felt systemically unwell, with a mild dry cough, a low grade fever, lethargy and malaise. Further investigation showed her eosinophilia had increased. The diagnosis was confirmed by a serum sample sent to the School of Hygiene and Tropical Medicine in London. A working diagnosis of filariasis was made. Filarial worms were present in the serum.

Filarial infections are common in Africa, Latin America, the Mediterranean and Asia. They are caused by parasitic filarial worms, which dwell in the tissue and are transmitted by mosquitoes and other biting insects. There are two main groups of filarial infections; lymphatic and subcutaneous types. ${ }^{1}$ The filarial worm genus Wuchereia has only one species, Bancrofti. The life cycle was first worked out by Sir Patrick Manson in $1878 .^{2}$ Microfilariae enter the mosquito's stomach and collect in the gut. At the end of 16 hours, a writhing mass is formed in the gut. The mosquito's proboscis then sucks up these microfilariae by positive chemotaxis and the proboscis is populated by a large concentration of microfilariae. The mosquito on landing on human skin bites in to the flesh and abstracts a cubic millimetre of blood at each feed and in doing this concentrates the embryos in the blood meal. They then enter the mosquito's thorax where they lie between the muscular fibres. Within two days the girth of the microfilariae increases and they assume a squat sausage shape form. Three papillae develop on the larvae that help ease the penetration through human skin. On the 10th day, the larval filarial, travels forward to the mosquito's head. At high temperatures and in a humid environment the complete cycle takes 10 to 14 days but can be retarded to six weeks in the cold. When an infected mosquito bites humans the larvae (attracted to the warmth) puncture the mosquito's proboscis and wriggle out onto the skin, which they penetrate near puncture sites caused by the stylets of the mosquito. In the human host the infective larvae pass through peripheral blood vessels to the lymphatics maturing in approximately three months to one year. Humans are the only known definitive host. Once in the serum, a lymphatic filariasis may occur with varying clinical features (fig 1).

In this case, it is most likely that our patient was exposed to infection in her later adult life and reacted more severely once she had come to live in a temperate climate found in the UK.

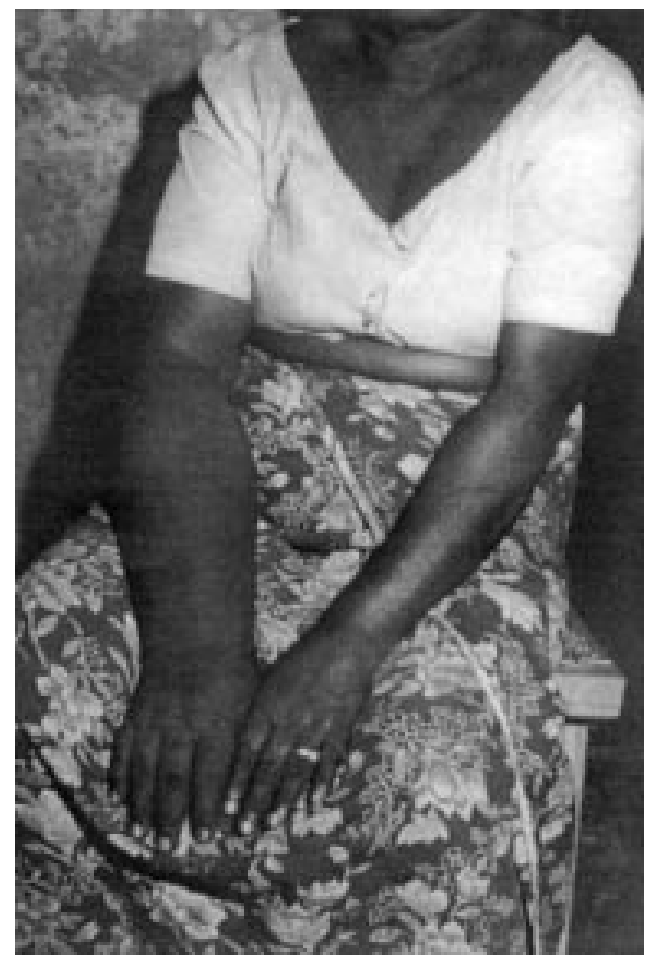

Figure 1 A typical presentation of lymphoedema by filariasis. 
Regarding lymphatic filariasis some patients only have microfilaraemia and others show an immunological response with positive filarial antigens. $^{3}$ There may be a filarial fever with recurrent episodes lasting three to seven days and be accompanied by a headache, malaise and adenolymphangitis. Repeated adenolymphangitis causes lymphoedema. If not treated severe elephantiasis will result. It is common in the lower limbs but can occur in the upper limbs as in this patient. The eosinophilia presents as a persistant, paroxysmal night-time cough and signs similar to bronchial asthma. This would explain the progression of the symptoms found in this case. This patient was treated with a course of diethylcarbamazine citrate, which kills the microfilariae and a proportion of the adult worms. This teatment proved very successful, and on follow up, she made an excellent recovery with no residual loss of function in her arm.

In conclusion, the duty doctor should have a high index of suspicion for a filarial worm infestation in patients presenting with swollen limbs with a history of travel in Africa, Asia, Latin America and the Mediterranean within the last year. The importance of a careful travel history cannot be overly emphasised.

Funding: none.

Conflicts of interest: none.

1 Duke BOL, Southgate BA. Filariasis. In: Weatherall DJ, Ledingham JGG, Warrell DA, eds. Oxford textbook of medicine. 3rd ed. Oxford: Oxford University Press, 1996: 7.14.1-2.

2 Manson-Bahr. Filariasis. Manson's tropical diseases. 18th ed. London: Baillere Tindall, 1990: 148-80.

3 Duke BOL. Filarial infections and diseases. Medicine Duke BOL. Filarial infect
International 1997;25:72.

\title{
An unusual cause of stridor
}

\author{
Paul Gaffney, Simon Holbrook
}

A 65 year old woman presented with acute stridor and breathlessness. She gave no prior history of respiratory symptoms and had no past medical history of note. Clinical examination was unremarkable with the exception of stridor and a moderate degree of respiratory distress. Her speech was normal. She had no palpable goitre. She was clinically and biochemically euthyroid.

Chest radiography revealed a superior mediastinal mass causing compression of the trachea and displacement to the right (fig 1). Subsequent mediastinal computed tomography (fig 2) confirmed the mass and suggested it as an extension of the left lobe of the thyroid gland. However, at operation a large ectopic goitre measuring $7.5 \times 4.5 \times 4 \mathrm{~cm}$ was resected

\section{Accident and Emergency \\ Department, St James's University Hospital, Leeds LS9 7TF \\ P Gaffney \\ S Holbrook \\ Correspondence to: Dr Gaffney}

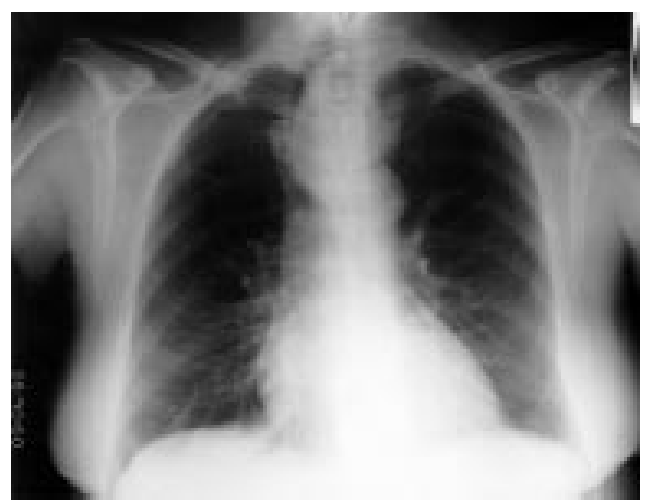

Figure 1 Mediastinal mass causing tracheal displacement and compression. by a cardiothoracic surgeon. It appeared that bleeding into the goitre had caused swelling and tracheal compression. The goitre was histologically benign.

Rarely, a mass in the anterior upper mediastinum may be a primary mediastinal goitre. ${ }^{1}$ Substernal extension is a well recognised feature of goitre arising in the anatomical thyroid gland and respiratory symptoms of a chronic, progressive nature are common in such cases. ${ }^{2}$ Sudden airway compromise caused by a goitre (ectopic or anatomical) rarely occurs but may necessitate immediate intubation. ${ }^{3}$

Funding: none.

Conflicts of interest: none.

1 Sand J, Pehkonen E, Matilla J, et al. Pulsating mass at the sternum: a primary carcinoma of ectopic mediastinal thyroid. F Thorac Cardiovasc Surg 1996;112:833-5.

2 Newman E, Shaha AR. Substernal goitre. F Surg Oncol 1995;60:207-12.

3 Shaha AR, Alfonso A, Jaffe BM. Acute airway distress due to thyroid pathology. Surgery 1987;102:1068-73.

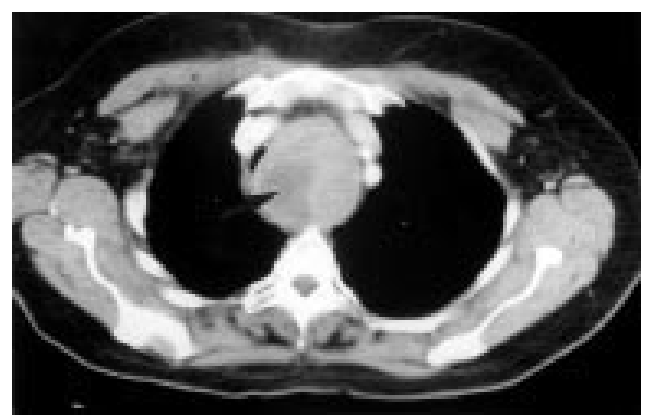

Figure 2 Comfirmatory evidence by computed tomography. 


\title{
A case of bilateral Lisfranc fracture-dislocations in a patient following polytrauma
}

\author{
M D Carter, M J Wilby
}

Department of

Trauma and

Orthopaedics, Ipswich

Hospital, Heath Road,

Ipswich, Suffolk

IP4 5PD

Correspondence to:

Dr Carter

(melaniecarter81@,

hotmail.com)

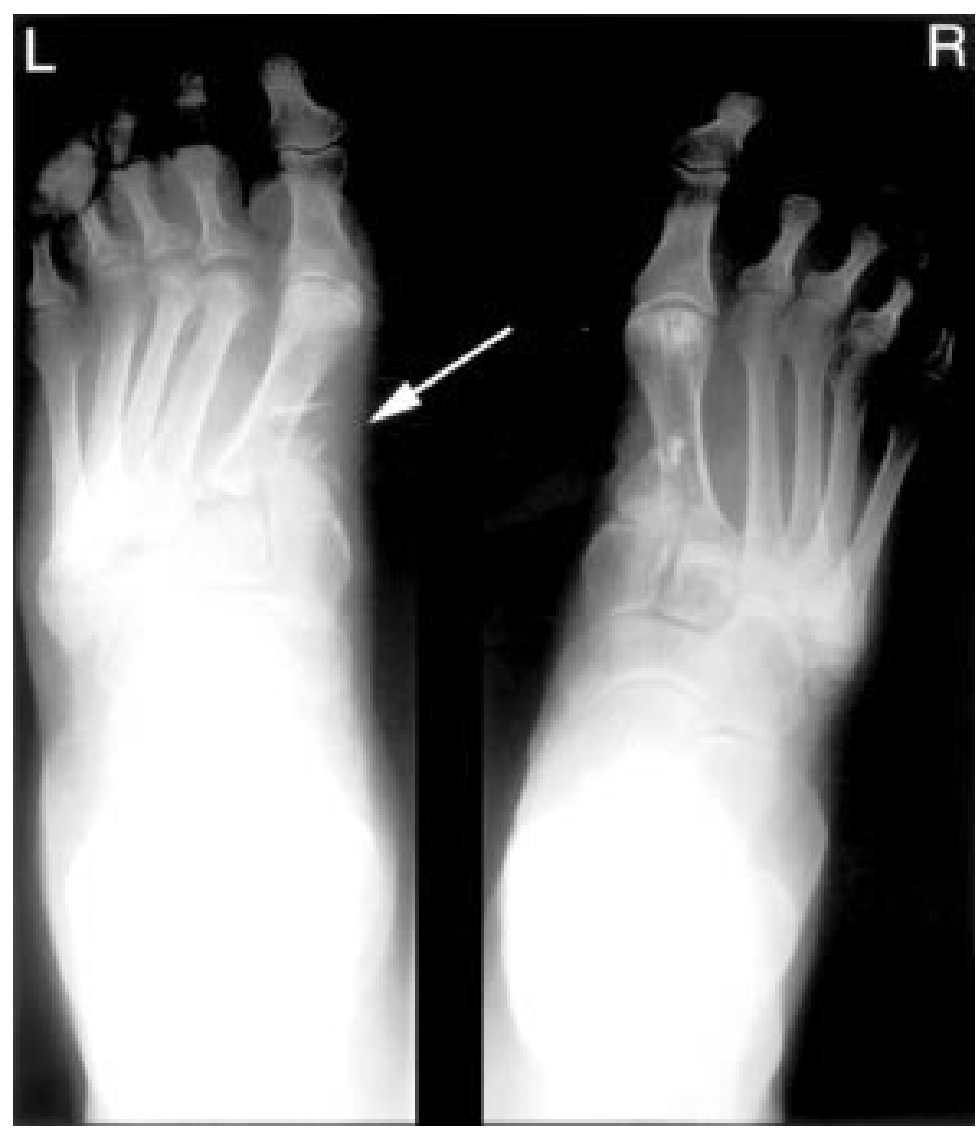

Figure 1 Anteroposterior radiographic views of the patient's feet at the time of admission The left hand panel represents the left foot.

A 64 year old woman was brought to the accident and emergency department after a head on road traffic accident with a combined speed of both cars approximating to $80 \mathrm{mph}$. The patient had not been able to free herself from the car and was amnesic for the events. Initial assessment revealed an alert and talking patient with grade II hypovolaemic shock.

\section{the} graphs fracture dislocations (fig 1). Closed reduction and internal fixation using Kirschner wires was performed. The patient made a good recovery from her injuries and was discharged two weeks later with continuing rehabilitation.

Lisfranc fractures have a reported incidence of 1 per 55000 people per year and a prevalence of $0.2 \%$ of all fractures. ${ }^{1}$ Some authors suggest that the "rarity" of these injuries may be attributable in part to doctors missing them, particularly in the context of polytrauma. $^{2}$ Overlooked Lisfranc fractures may lead to limb loss or more probably may predispose the patient to chronic pain and debilitation. This case illustrates how in a case of major trauma the initial examination may overlook the seemingly less important foot injuries and also the difficulty in clinically assessing painless symmetrical injuries. Moreover, this is a rare case of bilateral Lisfranc fractures, only previously reported bilaterally as occult injuries in non-trauma patients with diabetic neuropathic feet. ${ }^{3}$

Funding: none.

Conflicts of interest: none.

1 Hardcastle P, Neschauer R, Kutschu-Lisberg E, et al. Injuries to the tarso-metatrsal joint: incidence, classification and treatment. F Bone foint Surg 1982;64:349-56.

2 Englanoff G, Anglin D, Hutson HR. Lisfranc fracturedislocation: a frequently missed diagnosis in the emergency department. Ann Emerg Med 1995;26:229-33.

3 Hennessy O. Case report 264: Diabetic neuropathic disorder (osteoneuropathy) with bilateral Lisfranc fracturedislocations of feet. Skeletal Radiol 1982;11:155-7. 